\title{
Determination of microdroplet contact angles using electrically driven droplet oscillations
}

\author{
Y. Karadag, A. Jonáš, ${ }^{\text {a) }}$ N. Tasaltin, and A. Kiraz ${ }^{\text {b) }}$ \\ Department of Physics, Koç University, Rumelifeneri Yolu, 34450 Sariyer, Istanbul, Turkey
}

(Received 12 March 2011; accepted 15 April 2011; published online 9 May 2011)

\begin{abstract}
Oscillatory deformations of micrometer-sized $\mathrm{NaCl}$-water droplets by an $\mathrm{AC}$ electric field are used for contact angle measurements on superhydrophobic surfaces. Contact angles are determined from the dependence of the lowest-order resonant frequency of the electrically driven droplet oscillations on the droplet size. The resonant frequency and size of a droplet are found using whispering gallery mode spectroscopy. Measurements are compared with those performed with direct mechanical driving of the droplets using a piezoelectric transducer, and a good agreement is found. The demonstrated contact angle measurement method can be readily integrated into the planar architecture of microfluidic chips. (C) 2011 American Institute of Physics. [doi:10.1063/1.3588034]
\end{abstract}

Optical microcavities based on liquid microdroplets with readily tunable size, shape, and refractive index and exceptionally smooth surfaces can host high-quality optical resonances-the whispering gallery modes (WGMs). ${ }^{1}$ This property makes them well suited for on-chip integrated optofluidic applications, e.g., as reconfigurable optical switches, light sources, or chemical and biological sensors. ${ }^{2}$ Several strategies have been developed for incorporating the microdroplets into optofluidic systems. ${ }^{3}$ When dealing with droplets of aqueous (polar) liquids, it is advantageous to deposit them on a solid substrate with a superhydrophobic surface. ${ }^{4}$ Such a surface deposition stabilizes the position of the droplets without disturbing significantly their shape and, simultaneously, enables interfacing of the droplets with other components of a lab-on-a-chip device, such as integrated waveguides and electronics.

Recently, an application of liquid-based microcavities has been proposed that uses the analysis of sessile droplet vibrations for determining the liquid-solid contact angle $\theta$ over micrometer-sized contact areas. ${ }^{5}$ In this approach, surface-supported microdroplets were vibrated perpendicularly to the surface using a piezoelectric transducer (PZT) and the values of $\theta$ were subsequently calculated from the measured droplet mechanical resonant frequency and spherical radius using a previously developed theory of the sessile droplet axisymmetric oscillations. ${ }^{6}$ Vibrational resonances were identified by analyzing the spectral width of the WGMs present in the fluorescence spectra of the dye-doped microdroplets as a function of the droplet vibration frequency. Microscopic contact angle measurements based on the resonant frequency analysis were able to discriminate between surfaces with different superhydrophobicity.

In this paper, we extend the vibration-based contact angle measurement technique by introducing electric field driving of the microdroplet mechanical oscillations. To this end, the microdroplets are deposited in a channel formed between two planar gold electrodes on a superhydrophobic surface and subsequently excited by horizontal AC electric field applied between the electrodes (see Fig. 1). According to the leaky dielectrics model, this causes periodic droplet

\footnotetext{
a) Electronic mail: ajonas@ku.edu.tr.

${ }^{b)}$ Electronic mail: akiraz@ku.edu.tr.
}

deformations that are proportional to the square of the applied electric field. ${ }^{7,8}$ The use of horizontal AC electric field offers an alternative, more practical mechanism for exciting the droplet vibrations. Since the direct mechanical driving of the droplet-bearing substrate with a PZT is eliminated, substrate resonances do not influence the observed droplet mechanical response. Consequently, the droplet excitation is more uniform over the range of the driving frequencies used in the experiments.

The Strani-Sabetta theory (Ref. 6) describes axisymmetric vibrations of inviscid liquid droplets whose shape is completely determined by surface tension with a negligible influence of gravity. For the lowest-order vibration mode, the resonant frequency $f_{1}$ is given by $f_{1}=(2 \pi)^{-1}$ $\times\left[\gamma / \rho R^{3} \lambda_{1}(\theta)\right]^{1 / 2}$. Thus, for a droplet of known surface ten-

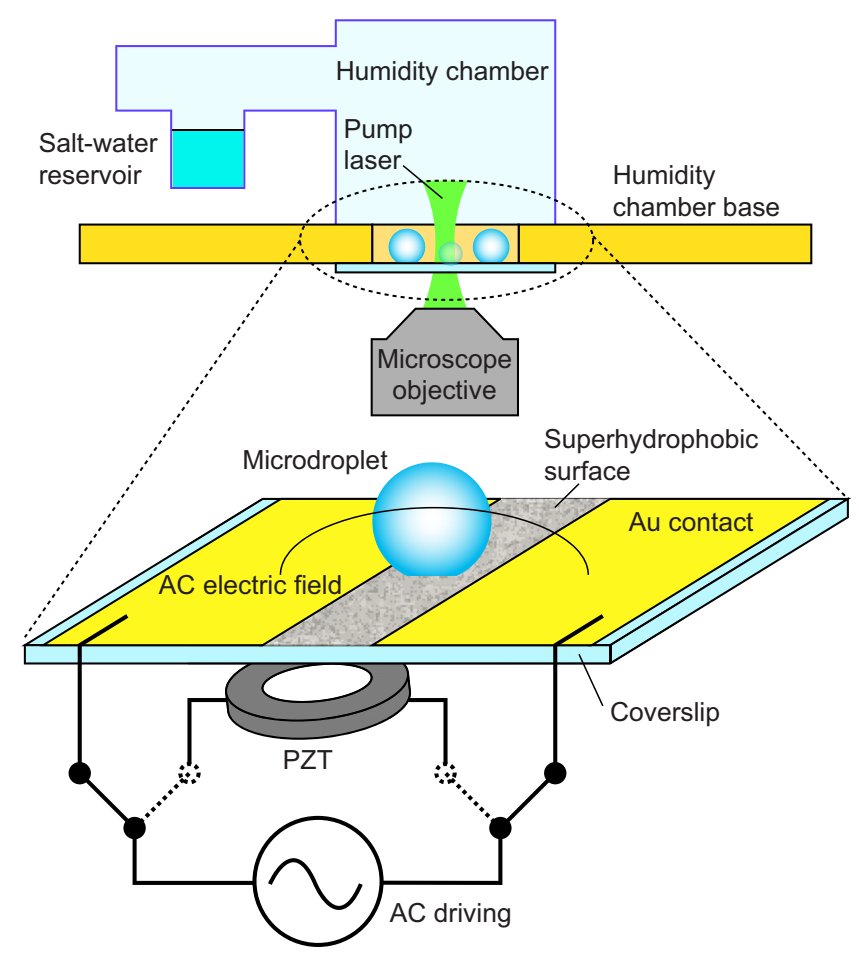

FIG. 1. (Color online) Experimental setup for contact angle measurements using vibrated microdroplets. Droplets are sequentially driven by a horizontal AC electric field and a PZT. 
sion $\gamma$, density $\rho$, spherical radius $R$, and resonant frequency $f_{1}$, the contact angle $\theta$ can be determined from the lowest-mode eigenvalue $\lambda_{1}(\theta)$ calculated from the above equation. ${ }^{6,9,10}$ Strictly speaking, driving the droplet oscillations with the horizontal AC electric field does not induce purely axisymmetric vibration modes as the driving force is perpendicular to the axis of symmetry of a sessile droplet. However, we show here that in the limit of small nonaxisymmetric deformations and large contact angles, droplet resonant frequencies determined sequentially using PZT (axisymmetric) and horizontal field (non-axisymmetric) driving are very close. Hence, the use of the Strani-Sabetta theory is justified for the contact angle measurements based on electrically driven droplet oscillations, reported in this paper.

We note that electrically driven oscillations in the horizontal electric field geometry have been previously reported for characterizing the mechanical frequency response of millimeter-sized water droplets. ${ }^{11}$ In that paper, however, large droplet deformations were observed that led to significant changes in the droplet contact angle during its oscillation. Also, droplets having relatively small contact angles $\left(\theta<124^{\circ}\right)$ were studied and no quantitative comparison to a theoretical model was made.

Our experimental setup is depicted in Fig. 1. Planar electrodes are prepared by subsequent evaporation of Ti (thickness: $0.5 \mu \mathrm{m}$ ) and $\mathrm{Au}$ (thickness: $1 \mu \mathrm{m}$ ) on coverglass substrates in a $10^{-5} \mathrm{~Pa}$ vacuum chamber. A copper mask is used during evaporation in order to obtain 70-90 $\mu \mathrm{m}$-wide insulating channels between the electrodes. Superhydrophobic surfaces are prepared by spin coating a suspension of hydrophobic silica nanoparticles $(50 \mathrm{mg}$ Aeroxide LE1 in $1 \mathrm{~mL}$ ethanol, average particle size: $14 \pm 3 \mathrm{~nm}$; Evonik) on coverglasses with electrodes. ${ }^{12}$ Rhodamine B-doped $\mathrm{NaCl}$-water microdroplets (2.5 M NaCl, $100 \mu \mathrm{M}$ Rhodamine B) are then sprayed into the channel between the Au electrodes by an ultrasonic nebulizer in ambient atmospheric conditions. A PZT is glued beneath the coverglasses for direct mechanical excitation of the droplet vibrations. Prepared coverglasses are then attached to the base of a sealed humidity chamber whose relative humidity is fixed at $84 \%$ with a saturated water solution of $\mathrm{KCl}$. Experiments are performed approximately one hour after sealing the chamber, once the droplets reach their equilibrium sizes.

WGMs of dye-doped droplets are observed using fluorescence spectroscopy. ${ }^{5,12}$ Individual droplets are excited in the vicinity of their rim by a $532 \mathrm{~nm} \mathrm{CW}$ green laser (maximal power $4.5 \mathrm{~mW}$ ) that is focused by an air objective $(\mathrm{NA}=0.8,60 \mathrm{x})$. The fluorescence emission is collected using the same objective and dispersed by a $500 \mathrm{~mm}$ monochromator with a $1200 \mathrm{gr} / \mathrm{mm}$ grating (spectral resolution: 0.07 $\mathrm{nm})$. A cooled charge coupled device camera is used for recording fluorescence spectra with an exposure time of 750 ms. High voltage oscillatory driving of the electrodes or the PZT is achieved by a high voltage amplifier attached to the output of a function generator, providing $\sim 300 \mathrm{~V}$ peak-topeak sinusoidal voltage at varying frequencies. During the experiments, mechanical resonances are characterized using both the PZT and the horizontal electric field driving for each microdroplet. In both cases, consecutive fluorescence spectra are recorded as a function of the excitation frequency and the mechanical resonances are revealed by the analysis of the
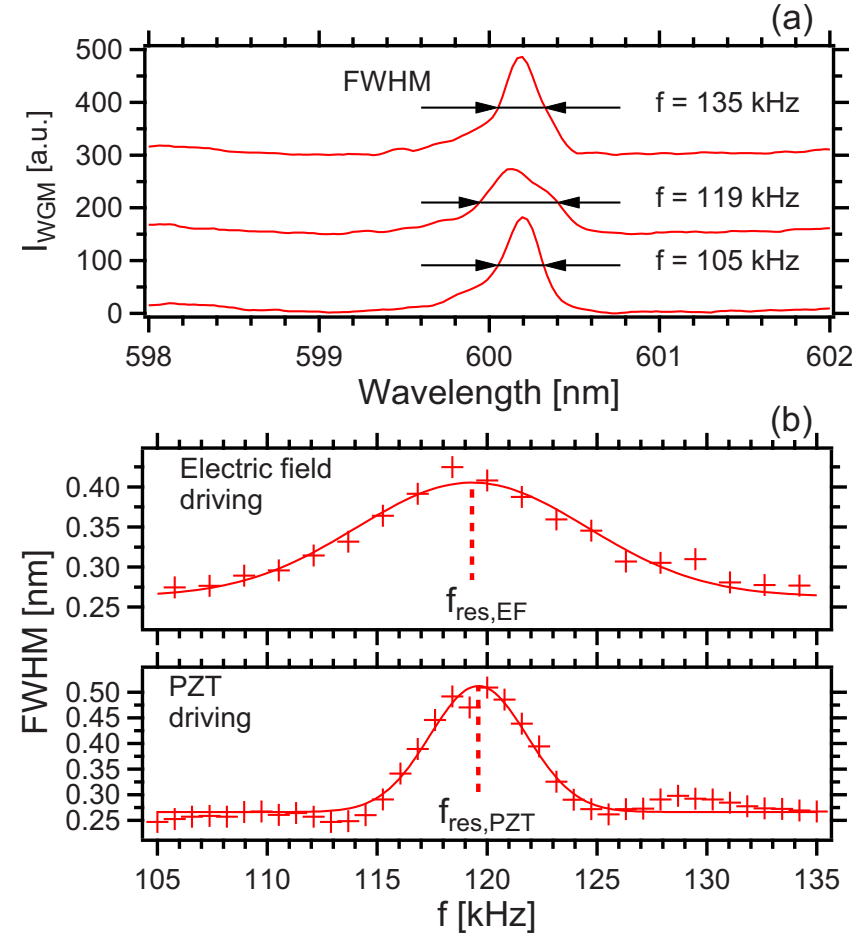

FIG. 2. (Color online) (a) Fluorescence spectra of a droplet with a spherical radius $R=5.4 \mu \mathrm{m}$ excited by a horizontal AC electric field at three different frequencies $f$. (b) FWHM of the WGM in (a) as a function of $f$ for horizontal electric field (top plot) and PZT (bottom plot) driving for the same droplet. Crosses indicate experimental data points, continuous lines are the Gaussian fits that reveal droplet resonant frequencies $f_{\text {res, } E F}^{\text {exp }}=119.3 \mathrm{kHz}$ and $f_{\text {res,PZT }}^{\text {exp }}=119.5 \mathrm{kHz}$, respectively.

frequency dependence of the WGM spectral widths. The droplet spherical radii are determined by the WGM modematching as described previously. ${ }^{5}$

Figure 2(a) shows exemplary fluorescence spectra recorded from a droplet with a spherical radius of $5.4 \mu \mathrm{m}$ upon horizontal electric field driving with frequencies of 105,119 , and $135 \mathrm{kHz}$. We note that, for the case of the horizontal electric field driving, excitation frequencies reported in this work are equal to twice the frequency of the AC voltage applied between the electrodes because of the quadratic dependence of the droplet deformation on the field strength. The WGM centered at $\sim 600.2 \mathrm{~nm}$ shows maximal broadening at $\sim 119 \mathrm{kHz}$ due to the mechanical resonance of the droplet. The mechanical resonant frequency is determined by analyzing the full width at half maximum (FWHM) of the WGM as a function of the excitation frequency [Fig. 2(b), top]. A Gaussian fit to the experimental data reveals a mechanical resonant frequency of $119.3 \mathrm{kHz}$ for this microdroplet. For comparison, Fig. 2(b), bottom, shows the frequency dependence of the FWHM of the same WGM obtained from the same microdroplet upon PZT driving. In this case, the Gaussian fit gives a mechanical resonant frequency of $119.5 \mathrm{kHz}$ which is very close to the value obtained by the electrical excitation. In general, near resonance, the mechanical frequency response of a droplet obtained with the electric field driving is broader than that obtained with the PZT driving. We attribute this mainly to the fact that, for the case of the PZT driving, the observed frequency response of the droplet is narrowed by multiplication of its actual frequency response by the spectrally non- 


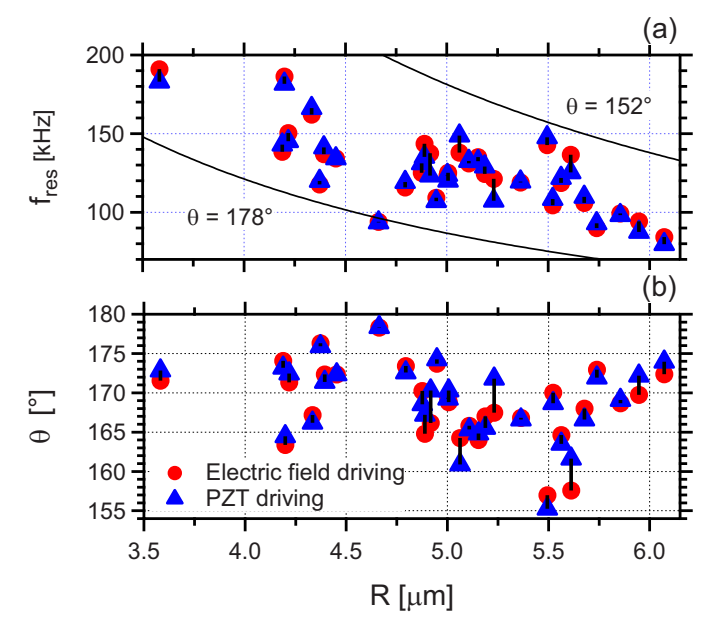

FIG. 3. (Color online) (a) Dependence of the measured mechanical resonant frequencies $f_{\text {res }}$ on the droplet spherical radius $R$. Circles and triangles indicate data points obtained with horizontal electric field and vertical PZT driving, respectively. Lines show the values of $f_{\text {res }}$ calculated using the Strani-Sabetta theory for contact angles of $178^{\circ}$ (lower frequency limit) and $152^{\circ}$ (upper frequency limit). Other calculation parameters for the $\mathrm{NaCl}$-water microdroplets: surface tension $\gamma=80.1 \mathrm{mN} / \mathrm{m}$, density $\rho$ $=1144.7 \mathrm{~kg} / \mathrm{m}^{3}$, and refractive index $n=1.3677$ (Refs. 12-14). (b) Contact angles calculated for the resonant frequencies in (a) using the Strani-Sabetta theory (Ref. 6).

uniform frequency response of the superhydrophobic substrate. ${ }^{5}$

Figure 3(a) shows the resonant frequencies of droplets with spherical radii varying between 3.5-6 $\mu \mathrm{m}$ obtained from sequential measurements performed using horizontal electric field (red circles) and PZT (blue triangles) driving. The contact angles calculated from the Strani-Sabetta theory for the experimental data presented in Fig. 3(a) are shown in Fig. 3(b). Only small relative differences between the horizontal electric field and PZT driving are observed in the measured resonant frequencies $(\max \pm 10 \%)$ and contact angles $(\max \pm 2 \%)$, with no systematic bias. These small differences that are mainly attributed to the substrate's nonuniform mechanical response during the PZT driving, indicate that in both cases, approximately axisymmetric vibrations predicted by the Strani-Sabetta theory are excited. This assumption is further justified by the relatively small $(<0.2 \%)$ non-axisymmetric deformations of the droplets due to the electric field intensities used for the droplet driving. ${ }^{15}$ Hence, the Strani-Sabetta theory is well suited for the contact angle measurements reported in this paper.

In conclusion, we have studied the lowest-order vibrational resonances of microdroplets on superhydrophobic sur- faces for droplet oscillations driven both horizontally with an AC electric field and vertically with a PZT. Our experimental results show that for small deformations, the horizontal electric field driving excites nearly axisymmetric droplet vibrational modes for contact angles $\theta>150^{\circ}$. Consequently, the theoretical model of the axisymmetric droplet oscillations can be used to calculate the droplet-surface contact angle. In comparison to the PZT driving, horizontal electric field driving offers a more practical mechanism for the contact angle measurement, enabling direct oscillatory excitation of the droplets, without being influenced by the substrate's mechanical response. Electric field driving can be applied to droplets immersed in another fluid instead of air. In such a situation, the amplitude of the driving will change depending on the material properties of both fluids (relative permittivity, conductivity, viscosity, and interfacial tension), ${ }^{7}$ but the general contact angle measurement procedure is not altered. Electric field driving can also provide an easy access to the selective excitation of chosen microdroplets using suitably designed electric contact geometries which can be integrated into microfluidic systems in a straightforward way.

This work is partially supported by TÜBITAK under Grant No. 109T734, European Commission Marie Curie IEF under Contract No. PIEF-GA-2009-252579 (A.J.), and FABED Young Investigator Research Award (A.K.).

${ }^{1}$ M. H. Fields, J. Popp, and R. K. Chang, in Progress in Optics, edited by E. Wolf (Elsevier, New York, 2000), Vol. 41, pp. 1-95.

${ }^{2}$ S. K. Y. Tang, R. Derda, Q. Quan, M. Loncar, and G. M. Whitesides, Opt. Express 19, 2204 (2011).

${ }^{3}$ D. Psaltis, S. R. Quake, and C. Yang, Nature (London) 442, 381 (2006).

${ }^{4}$ A. Kiraz, A. Kurt, M. A. Dündar, and A. L. Demirel, Appl. Phys. Lett. 89, 081118 (2006).

${ }^{5}$ A. Jonáš, Y. Karadag, N. Tasaltin, I. Kucukkara, and A. Kiraz, Langmuir 27, 2150 (2011).

${ }^{6}$ M. Strani and F. Sabetta, J. Fluid Mech. 141, 233 (1984).

${ }^{7}$ S. Torza, R. G. Cox, and S. G. Mason, Philos. Trans. R. Soc. London, Ser. A 269, 295 (1971).

${ }^{8}$ N. Bentenitis and S. Krause, Langmuir 21, 6194 (2005).

${ }^{9}$ R. W. Smithwick and J. A. M. Boulet, J. Colloid Interface Sci. 130, 588 (1989).

${ }^{10}$ R. W. Smithwick and J. A. M. Boulet, J. Colloid Interface Sci. 150, 567 (1992).

${ }^{11}$ T. Yamada, T. Sugimoto, Y. Higashiyama, M. Takeishi, and T. Aoki, IEEE Trans. Ind. Appl. 39, 59 (2003).

${ }^{12}$ A. Kiraz, Y. Karadag, S. C. Yorulmaz, and M. Muradoglu, Phys. Chem. Chem. Phys. 11, 2597 (2009).

${ }^{13}$ D. R. Lide, CRC Handbook of Chemistry and Physics (CRC, Cleveland, 2005); Internet Version 2005, http://www.hbcnetbase.com.

${ }^{14}$ Y. Marcus, J. Chem. Eng. Data 55, 3641 (2010).

${ }^{15}$ S. C. Yorulmaz, M. Mestre, M. Muradoglu, B. E. Alaca, and A. Kiraz, Opt. Commun. 282, 3024 (2009). 